\title{
Effect of larval crowding on mating competitiveness of Anopheles gambiae mosquitoes
}

Kija R Ng'habi*1,2, Bernadette John ${ }^{1,2}$, Gamba Nkwengulila², Bart GJ Knols ${ }^{3,4}$, Gerry F Killeen ${ }^{1,5}$ and Heather M Ferguson ${ }^{1,4}$

\begin{abstract}
Address: ${ }^{1}$ Ifakara Health Research and Development Centre (IHRDC), P. O. Box 53, Ifakara, Tanzania, ${ }^{2}$ University of Dar es Salaam, P. O. Box 35064 Dar es Salaam, Tanzania, ${ }^{3}$ International Atomic Energy Agency (IAEA), Agency's Laboratories Seibersdorf, Seibersdorf A-2444, Austria, ${ }^{4}$ Laboratory of Entomology. P.O. Box 8031, 6700 EH, Wageningen University, Wageningen, The Netherlands and ${ }^{5}$ Department of Public Health and Epidemiology, Swiss Tropical Institute, Basel, Switzerland

Email: Kija R Ng'habi* - kija@ifakara.mimcom.net; Bernadette John - bjohn@ifakara.mimcom.net; Gamba Nkwengulila - gamba@udsm.ac.tz; Bart GJ Knols - b.knols@iaea.org; Gerry F Killeen - gkilleen@ifakara.mimcom.net; Heather M Ferguson - Heather.Ferguson@wur.nl

* Corresponding author
\end{abstract}

Published: 30 September 2005

Malaria Journal 2005, 4:49 doi:10.1 186/1475-2875-4-49

This article is available from: http://www.malariajournal.com/content/4/1/49

(c) 2005 Ng'habi et al; licensee BioMed Central Ltd.

This is an Open Access article distributed under the terms of the Creative Commons Attribution License (http://creativecommons.org/licenses/by/2.0), which permits unrestricted use, distribution, and reproduction in any medium, provided the original work is properly cited.
Received: 17 August 2005

Accepted: 30 September 2005

\begin{abstract}
Background: The success of sterile or transgenic Anopheles for malaria control depends on their mating competitiveness within wild populations. Current evidence suggests that transgenic mosquitoes have reduced fitness. One means of compensating for this fitness deficit would be to identify environmental conditions that increase their mating competitiveness, and incorporate them into laboratory rearing regimes.
\end{abstract}

Methods: Anopheles gambiae larvae were allocated to three crowding treatments with the same food input per larva. Emerged males were competed against one another for access to females, and their corresponding longevity and energetic reserves measured.

Results: Males from the low-crowding treatment were much more likely to acquire the first mating. They won the first female approximately II times more often than those from the highcrowding treatment (Odds ratio $=1 \mathrm{I} . \mathrm{I}$ ) ) and four times more often than those from the mediumcrowding treatment (Odds ratio $=3.5 \mathrm{I}$ ). However, there was no overall difference in the total number of matings acquired by males from different treatments $(p=0.08)$. The survival of males from the low crowding treatment was lower than those from other treatments. The body size and teneral reserves of adult males did not differ between crowding treatments, but larger males were more likely to acquire mates than small individuals.

Conclusion: Larval crowding and body size have strong, independent effects on the mating competitiveness of adult male An. gambiae. Thus manipulation of larval crowding during mass rearing could provide a simple technique for boosting the competitiveness of sterile or transgenic male mosquitoes prior to release. 


\section{Background}

Mosquitoes within the Anopheles gambiae species complex are the most important vectors of malaria in sub-Saharan Africa [1-3]. The infective bite of these mosquitoes is in large part responsible for the more than 500 million clinical attacks of malaria reported worldwide each year, resulting in more than one million deaths $[4,5]$. Currently the two most widely implemented vector control strategies are indoor residual insecticide spraying and insecticide-treated bednets (ITNs), both of which have proven effective in the reduction of malaria transmission in some areas [6-11]. However, multiple insecticide resistance is emerging amongst the major malaria vectors An. gambiae [12] and Anopheles funestus [13], and there are complications associated with introduction, distribution and proper use of ITNs $[14,15]$ that indicate these strategies alone may not be sufficient to eliminate malaria transmission. New tools aimed at stopping malaria development in humans are promising, but the development of an efficacious antigen for vaccine production is slow, and parasite resistance to locally available drugs is increasing whilst new drugs that are effective are often unaffordable [16].

One promising new control prospect is the possibility of rendering wild vector populations less susceptible to infection by releasing mosquitoes that are genetically modified to resist infection [17-19], or sterile males that will mate with wild females and stop them from reproducing [20]. In the case of a genetically modified mosquito (GMM) strategy, malaria could be reduced by fixing a resistance gene in vector populations, [21-23], and in the case of sterile male release, malaria could be cut by a collapse in the vector population due to a high frequency of unviable matings. Any such release of sterile or GM mosquitoes should consist only of males [20,24] because this sex does not blood feed, and thus they will not increase the number or nature of mosquito bites per person at release sites. The success or failure of a GMM or sterile programme will depend largely on whether released males can successfully compete for mates against wild males $[25,26]$. Current evidence from laboratory experiments suggests that GMMs have reduced competitiveness and are generally out-competed in the presence of unmodified laboratory-reared males [27-29]. Operationally, the consequences of releasing males with poor competitiveness are dire. For example, the general failure of mosquito control programmes launched in the 1970s that aimed to reduce vector populations by releasing sterile males can be largely attributed to their poor mating competitiveness $[20,24]$, and to a lesser extent, the dispersal of fertile males into control areas. In the case of GMM, some argue that even if modified males have lower fitness than the wild type, refractory genes will still spread provided they are linked to an efficient genetic drive mechanism
[30]. However, such a drive mechanism could only act if insemination occurs in the first place [24], which it may not if GMM competitiveness is very low. Furthermore, no efficient genetic drive mechanism has yet been identified for Anopheles, and even assuming one is, there are doubts about whether it could be tightly linked to a potentially costly resistance gene $[23,31,32]$. The enhancement of male competitiveness thus remains crucial for successful gene introduction. Gaining an understanding of the ecological factors that govern Anopheles mating biology in general, and promote male competitiveness in particular, will increase the chances of success of future GMM and sterile male-based control efforts [24-26,33].

One ecological factor known to have a great influence on the life-history of adult Anopheline, Culicines and Aedes mosquitoes is the density at which larvae develop [34-37]. In nature, larvae of An. gambiae hatch and grow in a range of aquatic habitats [38]. In the absence of predators and pathogens, the number of larvae in a particular habitat and the amount of food available to them determines the number of adults that emerge from a habitat $[39,40]$, their survival $[37,40]$ and body size $[34,41,42]$. Crowded larvae are thought to be at a disadvantage because they are faced with greater competition for food [40], and are exposed to higher levels of toxic waste products, crowding chemicals and physical interference from other larvae [43-45].

Whereas the importance of larval density to female Anopheline and Aedes mosquitoes has been broadly investigated $[34,46,47]$, no doubt prioritized because of their direct role in disease transmission, little is known about its consequences for male mosquito vigour. Of the few known studies (in Anopheles and Aedes sp.) that have considered how larval density could influence male development $[41,48,49]$ their focus has been on the effect of food limitation, not that of chemical or physical interference.

Here the effect of larval crowding on the mating competitiveness of adult male An. gambiae was investigated. The focus was specifically on the effects of crowding in larval habitats, not on food limitation, which was controlled for by providing each larva with an equal amount of food per unit time. Crowding was prioritized for study because, space rather than food was believed to be the biggest limiting factor when mass-producing transgenic or sterile mosquitoes for field release. In addition to conducting mating assays, the teneral reserves of males from different crowding conditions was also quantified to test if any observed differences in competitiveness could be explained by energetic limitation. Energy reserves influence mosquito behavioural activities such as swarming and feeding [50,51], and may vary in response to larval crowding. In addition to testing the effect of larval crowding on mating competitiveness, it was also examined 
whether it influences male longevity, as this is another potential determinant of male lifetime reproductive fitness.

\section{Methods \\ Rearing}

An. gambiae sensu stricto from a colony at the Ifakara Health Research and Development (IHRDC), Tanzania, were used in this study. This colony was established from a wild population located near Njage village in 1996. First instar An. gambiae s.s. larvae were obtained from colony cages and assigned randomly to density treatments of 100 , 200 and 300 larvae per rearing tray $(37 \times 14 \times 13 \mathrm{~cm})$. Each tray was filled with $1 \mathrm{~L}$ of water and supplied with fish food $\left(\right.$ Tetramin $\left.^{\circledast}\right)$. In each tray, $0.2 \mathrm{mg}$ of Tetramin ${ }^{\circledast}$ was added for each larva, thus $20 \mathrm{mg}$, $40 \mathrm{mg}$ or $60 \mathrm{mg}$ was added to the low-, medium- and high-crowding treatment trays respectively, each day. Trays were inspected visually twice a day for the presence of pupae. Once detected, pupae were collected, counted and held individually in vials to allow for emergence. Batches of males from all three larval treatments that emerged on the same day were compared against one another in mating trials using females from the low-crowding treatment.

\section{Marking}

From the time of emergence, males were pooled according to crowding treatment and held in separate cages. On the second day after emergence, cohorts of adult males from two of the three rearing conditions were marked with green or pink fluorescent dusts respectively. One group was left unmarked. Marking treatments were alternated between crowding treatments across trials to ensure no systematic bias in performance due to dusting. Furthermore, pilot studies where males from the same crowding condition were marked with different colours revealed no effect of dust presence or colour on mating performance.

\section{Mating experiments}

On the third day after emergence, 30 males (10 males from each crowding treatment) were put together in one cage $(15 \times 15 \times 10 \mathrm{~cm})$. The cage was exposed to natural light a few hours before dusk. Observation of the cage began approximately 10 minutes prior to dusk. One or two males were observed to initiate the swarming process, just above a black disc (a swarm marker) that was placed on the bottom of the cage, with most of the remaining males joining the swarm after a few minutes. Once swarming was underway, 10 females from the low crowding condition were added to the cage (making a 3:1 male to female ratio). These females were simultaneously released into the cage using an aspirator. Mating activity was observed with a low-watt red light bulb. Pairs observed to form copula were immediately aspirated out of the cage and put together into a holding cup. On each evening of experiments, observation of mating was confined to an interval of 40-45 minutes. Observation ceased when all males had stopped swarming. At the close of the swarming session, unmated females were removed from the cage. The following morning, a fluorescent lamp was used to identify the larval rearing environment of each mated male.

Females observed to have copulated with males were blood-fed on the morning following mating and moved into individual vials. Wet filter paper was placed on the bottom of these vials to act as an oviposition site. After five days in individual holding tubes, all eggs laid by mated females were collected and counted. Wing lengths of both males and females that mated, as well as a subsample of those from males that did not, were measured under a dissecting microscope.

\section{Quantification of energy reserves}

Batches of newly emerged males from each larval crowding regime were killed by shaking and transferred individually into glass test tubes for the quantification of lipids, sugars and glycogen. Once in tubes, mosquitoes were crushed using a glass rod. One hundred micro-litres $(\mu \mathrm{l})$ of $2 \%$ sodium sulphate (which adsorbs glycogen) and $600 \mu \mathrm{l}$ of a 1:2 chloroform-methanol mixture (which dissolves lipids and sugars respectively) were added to each tube. Tubes were then covered and incubated for $24 \mathrm{hrs}$ at room temperature. For each batch of males that was analysed, one blank was prepared by adding the same chemicals to a tube that had no mosquito. Lipids, glycogen and sugars of each male, were then quantified using a colorimetric technique adapted for mosquito analysis [52].

\section{Longevity of unmated males}

In a separate series of experiments, males emerging from each larval rearing regime were denied access to females but held in cages to monitor their longevity. These males were provided with a $10 \%$ glucose solution for sustenance until death. All dead males were removed and counted daily.

\section{Statistical analyses}

The main aim of statistical analyses was to test for differences in the mating competitiveness, energy reserves, and longevity of An. gambiae males reared under different crowding conditions. Three analyses were conducted to assess mating competitiveness. First, analysis was restricted only to the first male to mate in each of 28 trials. The first male to mate was considered to be the fittest in the group (the first place 'winner'), and used a chi-square test to examine how larval crowding treatment influenced a male's probability of being a winner. Secondly, to test whether the total number of copulations in all nights was influenced by larval crowding treatment, a chi-square test 


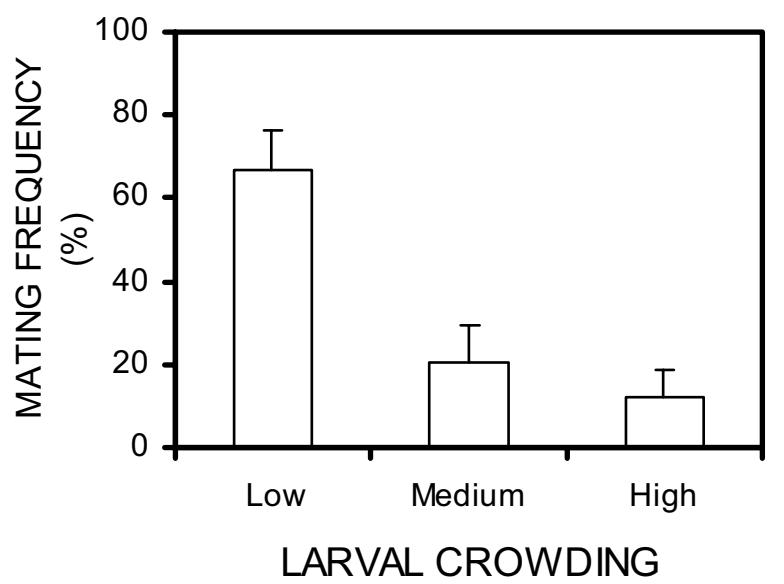

Figure I

Frequency at which males from high, medium and low crowding conditions were the 'first-to-mate' in 28 nights of mating trials. The error bars represent the standard error as estimated from the binomial distribution.

was again used. Finally, the order in which males mated during a night $\left(1^{\text {st }}, 2^{\text {nd }}, 3\right.$ rd etc. $)$ was examined whether was influenced by larval crowding treatment. For this, the analysis was restricted to data from the 14 trials (out of 28) where at least five matings occurred in a night. Males that mated were given a rank that corresponded to the order in which they mated during the trial (e.g. $1^{\text {st }}$ to mate got ' 1 ', etc). A Kruskal-Wallis test was then used to test the relationship between larval crowding treatment and mating rank (dependent variable). General Linear Models (GLM) were used to test whether larval crowding treatment influenced male wing length, or the abundance of lipids, glycogen and sugars they had on emergence. GLM were also used to test whether the number of eggs laid by a female was influenced by the larval crowding condition of the male that inseminated her. Finally, Kaplan-Maier survival analysis was used to test whether the survival of males depended on the crowding condition under which they were reared. All statistical analyses were done using the SPSS for windows and SAS system for Windows (version 8).

\section{Results}

\section{Mating competitiveness}

A total of 1,120 An. gambiae mosquitoes were used in 28 nights of mating experiments $(280$ females and 840 males). Restricting consideration to the first male to mate, we observed that males from low crowding environments were much more likely to succeed $\left(\chi_{2}{ }^{2}=13.61, \mathrm{p}=0.01\right.$,

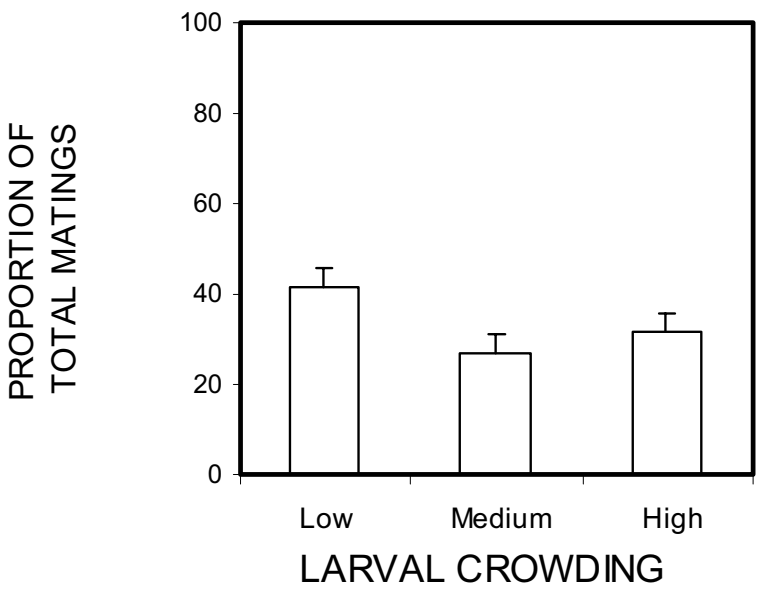

Figure 2

Proportion of total matings in 28 nights of trials going to males from low, medium and high larval crowding treatments. Error bars are the standard error as estimated from the binomial distribution $(n=133)$.

Figure. 1). Males from the low crowding treatment won approximatelly11 times (Odds ratio [95\% CI] $=11.17$, [2.7-50]) more often than those from the high crowding treatment, while those from the medium crowding condition won approximately 4 times more often (O.R [95\% $\mathrm{CI}]=3.51,[0.9-16.7]$ ). Analysis of all copulations (not just the first) in all 28 nights trials showed no statistically significant difference in mating frequency between males from different crowding treatments $\left(\chi_{2}{ }^{2}=4.99, \mathrm{p}=0.08\right)$, however there was a trend towards a higher mating frequency at low crowding condition, similar to that demonstrated in the 'first-to-mate' analysis (Figure 2). In the subset of 14 trials where at least five males mated, there was a weak tendency for males from the least crowded larval condition to mate before those from more crowded conditions, but it was not statistically significant $\left(\chi^{2}{ }_{2}=\right.$ 5.09, $\mathrm{p}=0.08$, Figure. 3 ).

The average size of male mosquitoes did not vary significantly between larval crowding treatments $\left(\mathrm{F}_{2,397}=2.43, \mathrm{p}\right.$ $=0.09$, mean body size: $2.75 \pm 0.23 \mathrm{~mm}, 2.79 \pm 0.12 \mathrm{~mm}$ and $2.79 \pm 0.12 \mathrm{~mm}$ for low-, medium- and higher-crowding conditions, respectively). However, of those that were measured ( $\mathrm{n}=398)$, males who successfully obtained a female were larger than those that did not $\left(\mathrm{F}_{1,397}=6.97, \mathrm{p}\right.$ $=0.01$, mean body size: $2.82 \pm 0.02 \mathrm{~mm}$ and $2.76 \pm 0.01$ $\mathrm{mm}$ respectively, Figure. 4). There was no difference between the body size of males who mated first, and those who mated later in the evening $\left(F_{1,115}=1.79, p=0.18\right.$, but 


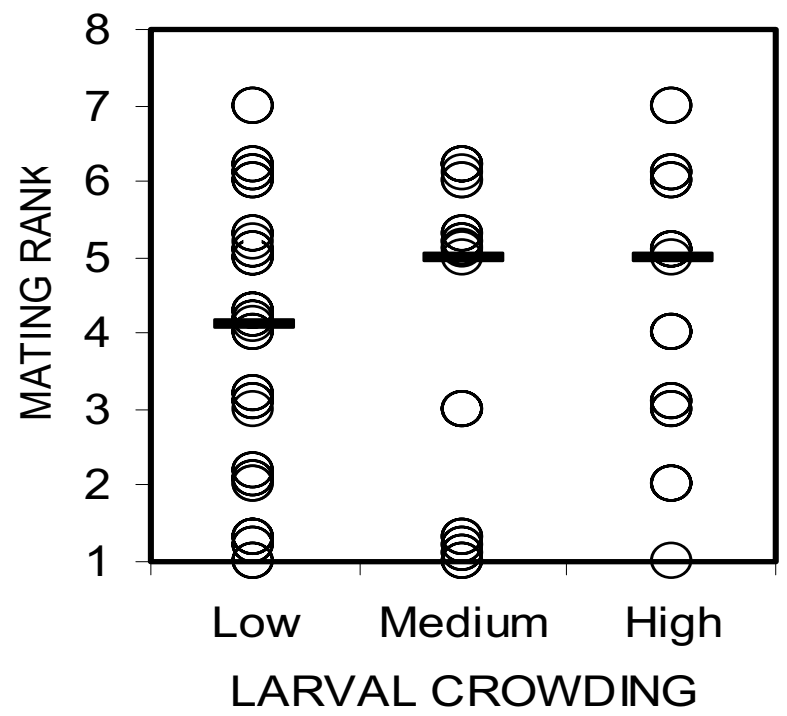

Figure 3

Distribution of mating ranks of males from low, medium and high crowding treatments as observed in the 14 mating trials in which at least 5 matings occurred. One circle represents two observations and the dark line in each treatment gives the median mating rank. Overlapping lines have the same mating rank, but have been spaced to indicate the number of observations per rank.

both groups were larger than males who did not mate, Figure. 4).

Only 15 out of 52 mated and subsequently blood-fed females oviposited their eggs. Amongst this subset, no association was found between egg batch size and paternal crowding condition $\left(\mathrm{F}_{2,12}=0.67, \mathrm{p}=0.53\right)$ or maternal wing length $\left(\mathrm{F}_{1,11}=1.98 \mathrm{p}=0.19\right)$. Additionally, there was no association between the probability that females would oviposit and the larval crowding condition of her mate $\left(\chi_{2}{ }^{2}=0.91, \mathrm{p}=0.63\right)$.

\section{Male teneral reserves and longevity}

Pooling all treatments, the mean amounts of teneral reserves in newly emerged males were $14.24( \pm 1.34) \mu \mathrm{g}$ of lipids, $1.34( \pm 0.71) \mu \mathrm{g}$ of sugars and $7.96( \pm 0.39) \mu \mathrm{g}$ of glycogen. There was no evidence that larval crowding conditions influenced the abundance of these reserves in newly emerged adult males (lipids: $\mathrm{F}_{2,66}=1.36, \mathrm{p}=0.26$, sugars: $\mathrm{F}_{2,66}=2.16, \mathrm{p}=0.12$ and glycogen: $\mathrm{F}_{2,66}=2.12, \mathrm{p}$ $=0.13$, Figure. 5).

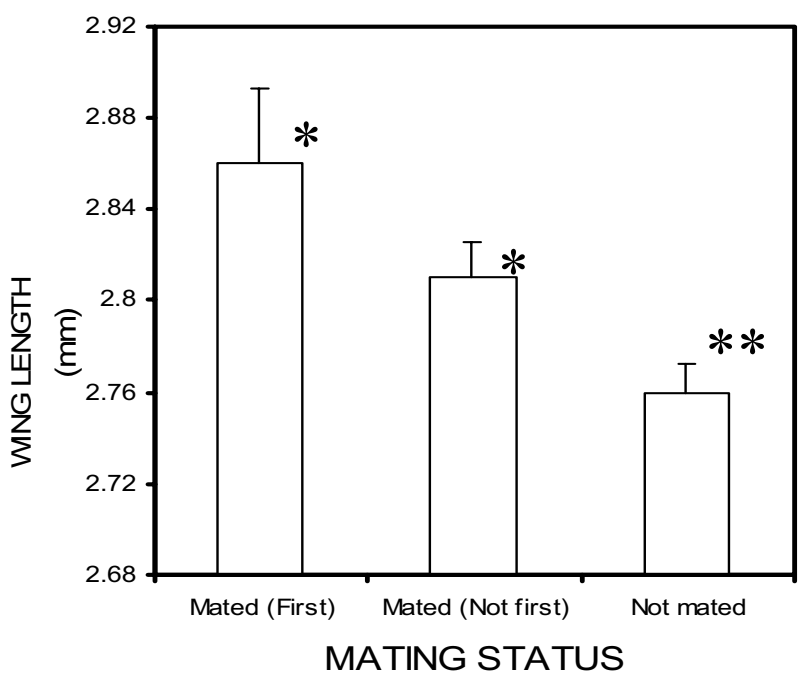

Figure 4

Body sizes (as indexed by wing length) of males who were the first to mate, who mated but were not the first, and that did not mate at all. Bars with the same number of asterisks (*) are not statistically different, but bars with differing numbers are.

The survival of 132 male An. gambiae was observed $\left(\mathrm{N}_{\text {low }}\right.$ $=44, \mathrm{~N}_{\text {medium }}=37$ and $\mathrm{N}_{\text {high }}=51$ ). The survival of adult males varied in response to the crowding conditions under which they were reared (Log-rank $=10.79, \mathrm{df}=2, \mathrm{p}$ $<0.01$, Figure 6), with the median survival of males equaling 21, 25 and 26 days for low-, medium- and highcrowding conditions, respectively. Males from low larval crowding conditions had poorer survival than those from medium (Log-rank $=7.14$, df $=1, \mathrm{p}<0.01$ ) and higher crowding treatment (Log-rank 8.14, $\mathrm{df}=1, \mathrm{p}<0.01$ ). The survival of males from medium and higher crowding conditions did not differ (Log-rank $=0.12, \mathrm{df}=1, \mathrm{p}=0.73$ ).

\section{Discussion}

This study shows that larval crowding influences the mating competitiveness of male An. gambiae mosquitoes. Results from 28 replicated experiments indicates that males reared under low crowding conditions are eleven times more likely to be the first in a swarming group to obtain a female than those reared at high crowding conditions. However, when all matings were considered (not just the first in each night), there was no evidence that the frequency of copulations obtained by males varied in response to larval crowding conditions. 


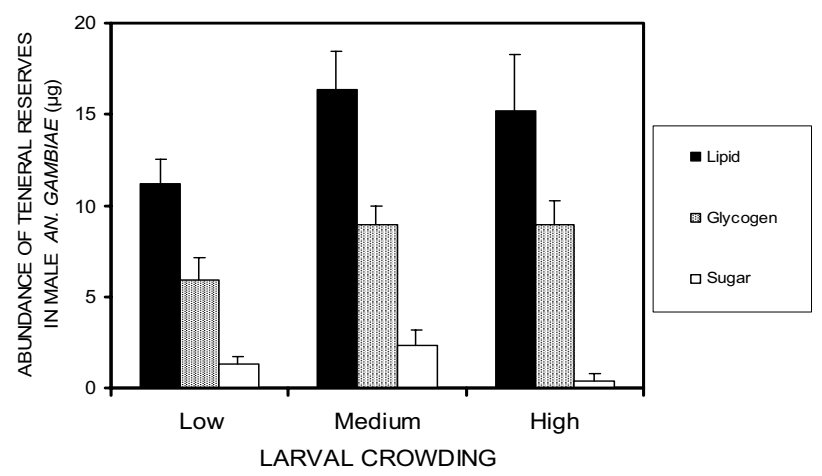

\section{Figure 5}

The mean mass of lipids, glycogen and sugar in newly emerged An. gambiae s. s. males reared in low, medium and high larval crowding conditions.

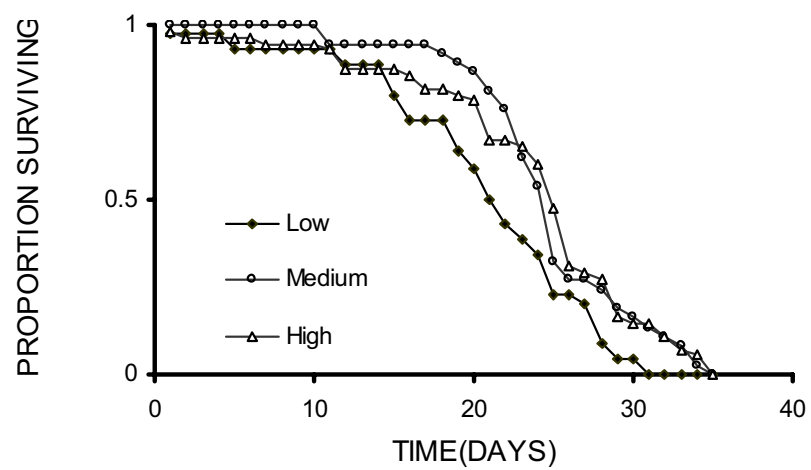

Figure 6

Survival of adult male An. gambiae s.s., from low, medium and high larval crowding treatments.

Thus, this study has shown that larval crowding conditions influences a male's chance of beating his competitors in order to obtain the first female, but not his chance of getting a female in general. What does this say about the role of larval crowding as a determinant of male fitness? It was proposed, that a male mosquito's ability to obtain the first available female is more likely to reflect their lifetime reproductive potential than their success in eventually getting a mate; especially under controlled laboratory conditions. There are several reasons for this hypothesis. The first is that during mating, male An. gambiae implant a mating plug in females which presents a temporary physical barrier to further insemination [5356]. Presuming females do not leave the swarm as soon as they are mated, males who hesitate may find themselves at a greater risk of encountering unreceptive females than those who mated first. Secondly, mating in An. gambiae is thought to be confined to a $15-20 \mathrm{~min}$ period [57-59] around dusk. Within this period, some males have been observed to return to the swarm after they have mated and continue seeking females [57]. Those who obtain the first females that enter the swarm are more likely to have sufficient time to return to the swarm after mating to look for additional females than those who mate later in the night. In our study, males were removed from the mating arena as soon as they obtained mate, so we could not test whether earlier maters were also more likely to mate repeatedly during the evening or not. However, this possibility is worth further study. A third reason for believing that those males who mated first have the highest mating competitiveness is that in nature, males are exposed to predation risks from insect predators, such as dragonflies, while swarming $[60,61]$. Those who mate first can leave the swarm and escape this risk, or even if they remain in the swarm, will have had the advantage of passing on their genes before being preyed upon. If predators we could have been introduced into the laboratory experimental cages, the ones that mated first might have exhibited an additional survival advantage. The final reason for hypothesizing that males who were the first to mate in our experiments would be the most competitive in nature is that the conditions under which male Anopheles compete for females in the field are much more intense than those created here. For example, while here the ratio created experimentally was 3 males to each female, in the field, males outnumber females at the mating site, in the range of $10: 1$ up to $600: 1[62,63]$. Under such skewed conditions where males dramatically outnumber females, it is extremely important for a male to seize a female at the earliest opportunity, as there is no guarantee another female will turn up before the end of the evening. Thus, any factor that was believed to increases a males chance of being the 'first-to-mate', as the study demonstrated with larval crowding, will be strongly correlated with their lifetime reproductive successes under natural conditions.

Several possible mechanisms that could explain the differences in mating patterns between crowding treatments were evaluated. The first was body size, which influenced the total number of males that mated, with larger males being more likely to obtain a mate than smaller ones. A similar finding was reported in Anopheles freeborni [57], whereas no size-dependency for mating was observed in An. gambiae by Charlwood [66]. Although body size influenced mating, in general it did not explain treatmentassociated differences in males who were the first-to-mate. This is because there was no difference in body size between males who mated first or later and no systematic difference in body size between crowding treatments. To conclude, both body size and larval crowding can inde- 
pendently influences male mating success, and that the effect of the latter is not exclusively driven by variation in the former trait.

The amount of teneral reserves in males did not differ between crowding treatments, and thus could not explain this differences in mating success. Eliminating these possibilities, it was hypothesized that the observed differences in mating success between crowding conditions could be due to the detrimental effects of chemicals $[43,45]$ and/or waste products that are released in crowded conditions, with larvae grown in dense conditions suffering more from exposure than those at low crowding.

When held at high density, some mosquito larvae release 'crowding chemicals' that retard the growth of their conspecifics [43]. This phenomenon has been recorded for Aedes aegypti, but not for Anopheles. In Aedes, chemical growth retardants are released by larvae when their density increases, even if each larva receives a constant ration of food [45]. There is, however, a certain food ration threshold above which no chemicals are produced regardless of the number of individuals $[44,45]$. When food rations are below this threshold, however, the release of these chemicals may regulate the number of adults that emerge [43]. Although the presence of such chemicals was not assayed here, its existence would explain why in the absence of food limitation, mosquitoes grown in highly crowded conditions performed poorer than those from low crowding. The mechanism through which such chemical factors could have influenced mating success is not clear, as it was not associated with between-treatment variation in body size or teneral reserves. Thus, it was assumed that exposure to these chemical factors may have led to subtle differences in size, behaviour or physiology not detected here (i.e. changes in male flight ability or reaction time) that ultimately influenced mating competitiveness. Further experiments are required to confirm whether such chemical factors exist in Anopheles, and how they operate.

Larval crowding also influenced the survival of An. gambiae adult males (Figure 4). Whereas males from low crowding conditions were generally the first to mate and, thus, probably the most competitive for mates, they also had the poorest survival. This observation suggests the existence of an energetic trade-off between reproduction and survival in male Anopheles, such as has been observed in other insects [64]. In male An. gambiae, such a trade-off could arise because males that are the first to mate are those that are the most active, and spend more time flying and swarming than those with lower mating success. As flying is energetically costly $[50,53]$, an increased tendency to do so may lead to both; an enhanced mating competitiveness and reduced long-term survival, as we observed in males from the low crowding condition here. As the proportion of time that males from different crowding conditions were flying in this experiment was not observed, it remains unknown if differential activity could explain the between-group variation in mating success and survival. Further study is required to measure whether flight activity is linked to mating success, and it whether influences the rate at which a male's energy reserves and longevity decrease.

The reduced survival of males from low crowding conditions may not necessarily compromise their long-term reproductive fitness. The benefits of being the first to mate during the early part of their adult life, as discussed above, may compensate for having a reduced number of mating opportunities in the longer term due to poorer survival. If so, our findings are consistent with the theoretical claim that longevity may not be a reliable measure of male reproductive fitness [64,65]. Further experiments in which males are given multiple opportunities to mate during their natural life are required to confirm whether being the first to mate on any given evening is indeed the best predictor of male mosquito lifetime reproductive success. Ideally these experiments would be carried in larger semi-field systems [33], as well as in natural populations, so realistic costs of activity (i.e. exposure to predation, energetic drain) can be incorporated.

\section{Conclusion}

These novel findings have direct application to genetic control strategies for malaria that seek to reduce transmission by releasing sterile or malaria-refractory Anopheles males. The reported poor competitive success of transgenic male mosquitoes [27-29] could be enhanced by rearing males in conditions of low crowding and high food abundance. This could create a cohort of highly competitive yet relatively short-lived males for release. Ideally, transgenic males should be both highly competitive and long-lived. However, should an energetic trade-off exist between their competitiveness and longevity as suggested here, we argue it would be more useful to focus on increasing their short-term mating competitiveness by methods such as those discussed here.

To increase the competitiveness of mass-reared males, it is advocated: 1) to maintain males at low densities and/or regular changing of rearing water to avoid the build-up of crowding of chemicals that might result in disadvantaged males, and 2) to supply larvae with sufficient amounts of food. This finding, therefore, may help to overcome some of the mating-related hurdles that impeded early genetic control trials [24]. This proposes that, the fitness of all current genetically modified Anopheles constructs $[17,19]$ be re-assayed after under ideal larval conditions in order to 
show how substantially ecological manipulation could increase their mating success relative to the wild type.

\section{Authors' contributions}

$\mathrm{KN}$ and $\mathrm{BJ}$ were directly involved in the experimental work. KN, HF and GK developed the experimental design. HF helped in logistics, advised statistical analysis of the data and supervised manuscript preparation. BGJK obtained funding for this work. GK, BGJK and GN provided comments on the manuscript prior to submission.

\section{Acknowledgements}

We would like to thank the entomology team at the IHRDC for their invaluable assistance. We thank Dr. Ana Rivero for her advice regarding energy reserve analysis, and Dr. Tom Smith and Dr. Nick Colegrave for discussion of statistical analysis. We are also thankful to Dr. Mark Benedict for helpful comments during manuscript preparation. This research is funded by a VIDI grant (no. 864.03.004) awarded by the Dutch Scientific Organisation (NWO) to Bart G.J. Knols, and carried out at the IHRDC.

\section{References}

I. White GB: Anopheles gambiae complex and disease transmission in Africa. Trans R Soc Trop Med Hyg 1974, 68:279-30I.

2. Gillies MT, DeMeillon B: The Anophelinae of Africa South of the Sahara (Ethiopian zoogeographical region) Volume 54. Johannesburg: South African Institute for Medical Research; 1968.

3. Kiszewski A, Mellinger A, Spielman A, Malaney P, Sachs SE, Sachs J: A global index representing the stability of malaria transmission. Am J Trop Med Hyg 2004, 70:486-498.

4. Hay IS, Guerra AC, Tatem JA, Atkinson MP, Snow WR: Urbanization, malaria transmission and disease burden in Africa. Nature Rev 2005, 3:8I-90.

5. Snow RW, Guerra CA, Noor AM, Myint HY, Hay SI: The global distribution of clinical episodes of Plasmodium falciparum malaria. Nature 2005, 434:2I4-217.

6. Alten B, Caglar SS, Simsek FM, Kaynas S: Effect of insecticidetreated bednets for malaria control in Southeast AnatoliaTurkey. J Vect Ecol 2003, 28:97-107.

7. Hawley WA, ter Kuile FO, Steketee RS, Nahlen BL, Terlouw DJ, Gimnig JE, Shi YP, Vulule JM, Alaii JA, Hightower AW, Kolczak MS, Kariuki SK, Phillips-Howard PA: Implications of the western Kenya permethrin-treated bed net study for policy, programme implementation, and future research. Am J Trop Med Hyg 2003, 68:168-173.

8. Wiseman V, Hawley WA, ter Kuile FO, Phillips-Howard PA, Vulule JM, Nahlen BL, Mills AJ: The cost-effectiveness of permethrintreated bed nets in an area of intense malaria transmission in western Kenya. Am J Trop Med Hyg 2003, 68:16I-I67.

9. ter Kuile FO, Terlouw DJ, Phillips-Howard PA, Hawley WA, Friedman JF, Kolczak MS, Kariuki SK, Shi YP, Kwena AM, Vulule JM, Nahlen $B L$ : Impact of permethrin-treated bed nets on malaria and all-cause morbidity in young children in an area of intense perennial malaria transmission in western Kenya: cross-sectional survey. Am J Trop Med Hyg 2003, 68:100-107.

10. Curtis CF: Should the use of DDT be revived for malaria vector control? Biomedica 2002, 22:455-46I.

II. Roberts DR, Manguin S, Mouchet J: DDT house spraying and reemerging malaria. Lancet 2000, 356:330-332.

12. Yawson AE, McCall PJ, Willson MD, Donnelly MJ: Species abundance and insecticide resistance of Anopheles gambiae in selected areas of Ghana and Burkina Faso. Med Vet Entomol 2004, I 8:372-377.

13. Brooke BD, Kloke G, Hunt RH, Koekemoer LL, Temu EA, Taylor ME, Small G, Hemingway J, Coetzee M: Bioassay and biochemical analyses of insecticide resistance in southern African Anopheles funestus (Diptera: Culicidae). Bull Entomol Res 2001, 91:265-273.

14. Curtis C, Maxwell C, Lemnge M, Kilama WL, Steketee RW, Hawley WA, Bergevin Y, Campbell CC, Sachs J, Teklehaimanot A, Ochola S, Guyatt H, Snow RW: Scaling-up coverage with insecticide- treated nets against malaria in Africa: who should pay? Lancet Infect Dis 2003, 3:304-307.

15. Lengeler C: Insecticide-treated bed nets and curtains for preventing malaria. Cochrane Database Syst Rev 2004, 2:.

16. WHO/UNICEF: The African Malaria Report 2003. The African Malaria Report 2003:I20.

17. Ito J, Ghosh A, Moreira LA, Wimmer EA, Jacobs-Lorena M: Transgenic anopheline mosquitoes impaired in transmission of a malaria parasite. Nature 2002, 4I 7:452-455.

18. Moreira AL, Ito J, Ghosh A, Devenport M, Zieler H, Abraham EG, Crisanti A, Nolan T, Catteruccia F, Jacobs-Lorena M: Bee venom phospholipase inhibits malaria parasite development in transgenic mosquitoes. J Biol Chem 2002, 277:40839-40843.

19. James AA: Blocking malaria parasite invasion of mosquito salivary glands. J Exp Biol 2003, 206:3817-382I.

20. Benedict $M Q$, Robinson AS: The first releases of transgenic mosquitoes: an argument for the sterile insect technique. Trends Parasitol 2003, I 9:349-355.

21. Kiszewski AE, Spielman A: Spatially explicit model of transposon-based genetic drive mechanisms for displacing fluctuating populations of anopheline vector mosquitoes. J Med Entomol 1998, 35:584-590.

22. Mshinda H, Killeen GF, Mukabana WR, Mathenge E, Mboera LEG, Knols BGJ: Development of genetically modified mosquitoes in Africa. Lancet Infect Dis 2004, 4:264-265.

23. Tabachnick WJ: Reflections on the Anopheles gambiae genome sequence, transgenic mosquitoes and the prospect for controlling malaria and other vector borne diseases. J Med Entomol 2003, 40:597-606.

24. Ferguson FM, John B, Ng'habi K, Knols BGJ: Addressing the sex imbalance in knowledge of vector biology. Trends Evol Ecol 2005, 20:202-209.

25. Knols BG Jr, Njiru BN, Mathenge EM, Mukabana WR, Beier JC, Killeen GF: Malariasphere: A greenhouse-enclosed simulation of a natural Anopheles gambiae (Diptera: Culicidae) ecosystem in Western Kenya. Malar J 2002, I: 19.

26. Scott TW, Takken W, Knols BG Jr, Boete C: The ecology of genetically modified mosquitoes. Science 2002, 298: I I7- II9.

27. Catteruccia F, Godfray HC, Crisanti A: Impact of genetic manipulation on the fitness of Anopheles stephensi mosquitoes. Science 2003, 299: I225-1227.

28. Irvin N, Hoddle MS, O'Brochta DA, Carey B, Atkinson PW: Assessing fitness costs for transgenic Aedes aegypti expressing the GFP marker and transposase genes. PNAS 2004, I $01: 891-896$.

29. Moreira LA, Wang J, Collins FH, Jacobs-Lorena M: Fitness of anopheline mosquitoes expressing transgenes that inhibit Plasmodium development. Genetics 2004, I 66: I337-I34|.

30. Boëte C, Koella JC: A theoretical approach to predicting the success of genetic manipulation of malaria mosquitoes in malaria control. Malar J 2002, I:3.

31. Gould F, Schliekelman P: Population genetics of autocidal control and strain replacement. Ann Rev Entomol 2004, 49:193-2I7.

32. Riehle MA, Srinivasan P, Moreira CK, Jacobs-Lorena M: Towards genetic manipulation of wild mosquito populations to combat malaria: advances and challenges. J Exp Biol 2003, 206:3809-3816.

33. Knols BG Jr, Njiru BN, Mukabana RW, Mathenge EM, Killeen GF: Contained semi-field environments for ecological studies on transgenic African malaria vectors. In Ecology of transgenic mosquitoes Edited by: Scott TW, Takken W. Wageningen: Wageningen University and Research Centre; 2003:99-106.

34. Lyimo EO, Takken W, Koella JC: Effect of rearing temperature and larval density on larval survival, age at pupation and adult size of Anopheles gambiae. Entomol Exp Appl 1992, 63:265-27I.

35. Koella JC, Lyimo EO: Variability in the relationship between weight and wing length of Anopheles gambiae. J Med Entomol 1996, 33:26I-264.

36. Agnew P, Haussy C, Michalakis Y: Effects of density and larval competition on selected life history traits of Culex pipiens quinquefasciatus (Diptera: Culicidae). J Med Entomol 2000, 37:732-735

37. Hawley $W$ : The effect of larval density on adult longevity of a mosquito, Aedes sierrensis : Epidemiological consequences. J Anim Ecol 1985, 54:955-964. 
38. Fillinger U, Sonye G, Killeen G, Knols BG Jr, Becker N: The practical importance of permanent and semipermanent habitats for controlling acquatic stages of Anopheles gambiae sensu lato mosquitoes: Operational observations from a rural town in western Kenya. Trop Med Inter Health 2004, 9: I274- 1289.

39. Gimnig JE, Ombok M, Kamau L, Hawley WA: Characteristics of larval anopheline (Diptera: Culicidae) habitats in Western Kenya. J Med Entomol 200I, 38:282-288.

40. Ye-Ebiyo Y, Pollack RJ, Kiszewski A, Spielman A: Enhancement of development of larval Anopheles arabiensis by proximity to flowering maize (Zea mays) in turbid water and when crowded. Am J Trop Med Hyg 2003, 68:748-752.

4I. Schneider JR, Morrison AC, Astete H, Scott TW, Wilson ML: Adult size and distribution of Aedes aegypti (Diptera: Culicidae) associated with larval habitats in lquitos, Peru. J Med Entomol 2004, 41:634-642.

42. Gama RA, Alves Kde C, Martins RF, Eiras AE, Resende MC: Effect of larvae density on adult size of Aedes aegypti reared under laboratory condictions. Revis Soc Bras Med Trop 2005, 38:64-66.

43. Roberts D: Overcrowding of Culex sitiens (Diptera: Culicidae) larvae: Population regulation by chemical factors or mechanical interference. J Med Entomol 1998, 35:665-669.

44. Reisen WK: Intraspecific competition in Anopheles stephensi Liston. Mosq News 1975, 35:470-480.

45. Moore CG, Whitacre DM: Competition in mosquitoes. 2. Production of Aedes aegypti larval growth retardant at various densities and nutritional levels. Ann Entomol Soc Am 1972, 65:915-918.

46. Mercer DR: Effects of larval density on the size of Aedes polynesiensis adults (Diptera: Culicidae). J Med Entomol 1999, 36:702-708

47. Armbruster $P$, Hutchinson RA: Pupal mass and wing length as indicators of fecundity in Aedes albopictus and Aedes geniculatus (Diptera: Culicidae). J Med Entomol 2002, 39:699-704.

48. Gomulski LM: Larval density, adult size and mating competitiveness in the mosquito Anopheles gambiae. Trans $R$ Soc Trop Med Hyg 1985, 79:276-277.

49. Koenraadt CJM, Takken W: Cannibalism and predation among larvae of the Anopheles gambiae complex. Med Vet Entomol 2003, I 7:6I-66.

50. Briegel $\mathrm{H}$ : Physiological bases of mosquito ecology. J Vect Ecol 2003, 28: I-II.

5I. Holliday-Hanson ML, Yuval B, Washino RK: Energetics and sugarfeeding of field-collected anopheline females. J Vect Ecol 1997, 22:83-89.

52. Van Handel E: Assay of lipids, glycogen and sugars in individual mosquitoes: correlations with wing length in field collected Aedes vexans. J Am Mosq contr Assoc 1988, 4:549-550.

53. Charlwood JD, Jones MDR: Mating behaviour in the mosquito, Anopheles gambiae s.l. Close range and contact behaviour. Physiol Entomol 1979, 4: I II-120.

54. Craig GB Jr: Mosquitoes: Female monogamy induced by male accessory gland substance. Science 1967, 156:1499-1501.

55. Bryan JH: Results of consecutive matings of female Anopheles gambiae Sp B with fertile and sterile males. Nature 1968, 21 8:489.

56. Giglioli MEC, Mason GF: The mating plug in anopheline mosquitoes. Proc R Soc London. Series A 1966, 41:123-129.

57. Yuval B, Wekesa JW, Washino RK: Effect of body size on swarming behaviour and mating success of male Anopheles freeborni (Diptera: Culicidae). J Insect Behav 1993, 6:333-342.

58. Charlwood JD, Pinto J, Sousa CA, Madsen H, Ferreira C, do Rosario VE: The swarming and mating behaviour of Anopheles gambiae s.s. (Diptera: Culicidae) from Sao Tome Island. J Vect Ecol 2002, 27:178-183.

59. Charlwood JD, Thompson R, Madsen H: Observations on the swarming and mating behaviour of Anopheles funestus from southern Mozambique. Malar J 2003, 2:2.

60. Downes JA: The swarming and mating flight of Diptera. Ann Rev Entomol 1969, | 4:27|-298.

61. Subramanian KA: Nature watch: When dragons fly. Resonance 2002:68-78.

62. Charlwood JD, Jones MDR: Mating behaviour in the mosquito, Anopheles gambiae s.l. II. Swarming behaviour. Physiol Entomol 1980, 5:315-320.
63. Reisen WK, Aslam Y, Siddiqui TF: Observation on the swarming and mating of some Pakistan mosquitoes in nature. Ann Entomol Soc Am 1977, 70:988-995.

64. Hunt J, Brooks R, Jonnions MD, Smith MJ, Bentsen CL, Bussiere LF: High-quality male field crickets invest heavily in sexual display but die young. Nature 2004, 432: 1024-1027.

65. Hansen TF, Price DK: Good genes and old age: Do old mates provide superior genes? J Evol Biol 1995, 8:759-778.

66. Charlwood JD, Pinto J, Sousa CA, Ferreira C, Do Rosario VE: Male size does not affect mating success of Anopheles gambiae in Sao Tome. Med Vet Entomol 2002, 16:109-1II.
Publish with BioMed Central and every scientist can read your work free of charge

"BioMed Central will be the most significant development for disseminating the results of biomedical research in our lifetime. "

Sir Paul Nurse, Cancer Research UK

Your research papers will be:

- available free of charge to the entire biomedical community

- peer reviewed and published immediately upon acceptance

- cited in PubMed and archived on PubMed Central

- yours - you keep the copyright

Submit your manuscript here:

http://www.biomedcentral.com/info/publishing_adv.asp
BiolMedcentral 